\section{Hepatic infarction caused by vascular migration of fiducial marker previously placed under endo- sonographic guidance to assist radiotherapy}

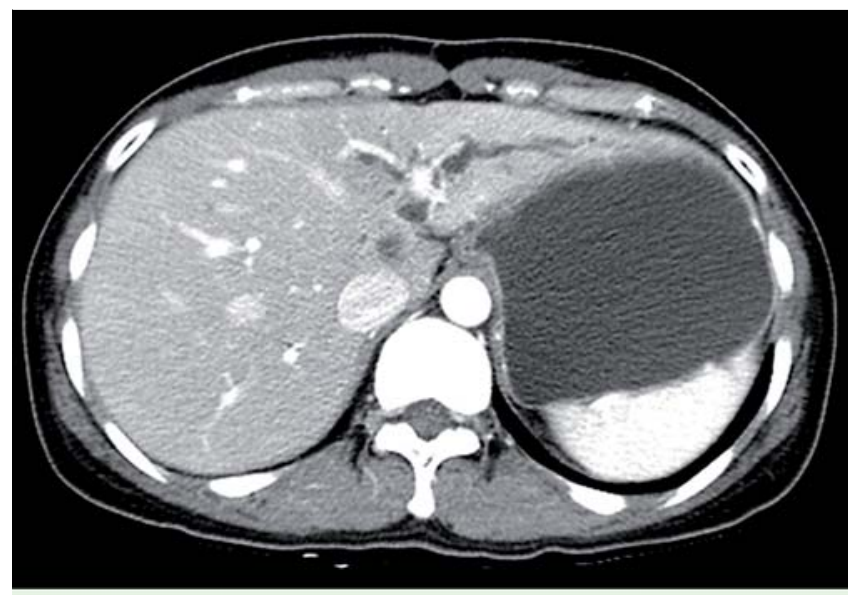

Fig. 1 A computed tomography (CT) scan from a 39-year-old woman with jaundice revealed a low attenuation $3.3 \times 2.0 \mathrm{~cm}$ caudate mass with left hepatic artery and left portal vein invasion, and bile duct obstruction.
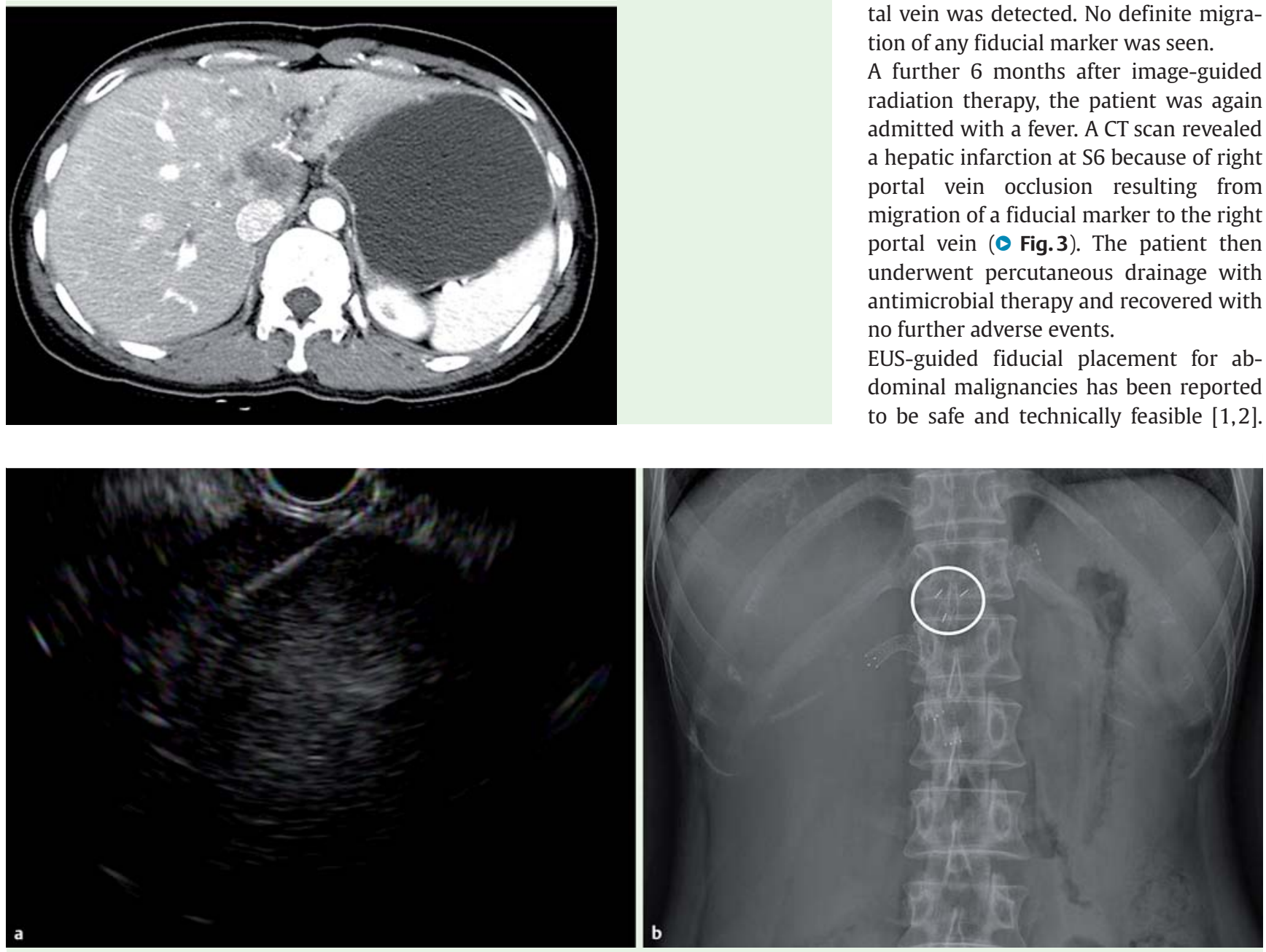

Fig. 2 a, b As the cholangiocarcinoma was unresectable, three fiducial markers were inserted into the tumor under endoscopic ultrasound (EUS) guidance.
A 39-year-old woman attended our hospital with jaundice. A computed tomography $(\mathrm{CT})$ scan revealed a low attenuation $3.3 \times 2.0 \mathrm{~cm}$ mass in the caudate lobe of the liver with left hepatic artery and left portal vein invasion, and bile duct obstruction ( $\bullet$ Fig. 1). Endoscopic biliary drainage and endoscopic ultrasound fineneedle aspiration (EUS-FNA) were performed. A biopsy taken from the mass contained adenocarcinoma cells. Because of the unresectability of this cholangiocarcinoma, three fiducial markers were inserted into the tumor under EUS guidance ( $\bullet$ Fig. 2). Image-guided radiation therapy was then performed with a $26-G y$ dosage delivered in four fractions. The patient also received systemic chemotherapy and photodynamic therapy. After 5 months, a follow-up CT scan showed that the tumor mass in the caudate lobe was markedly smaller. However, thrombosis of left and right (P7) branches of the portal vein was detected. No definite migration of any fiducial marker was seen.

A further 6 months after image-guided radiation therapy, the patient was again admitted with a fever. A CT scan revealed a hepatic infarction at $\mathrm{S} 6$ because of right portal vein occlusion resulting from migration of a fiducial marker to the right portal vein ( Fig.3). The patient then underwent percutaneous drainage with antimicrobial therapy and recovered with no further adverse events.

EUS-guided fiducial placement for abdominal malignancies has been reported to be safe and technically feasible $[1,2]$. 

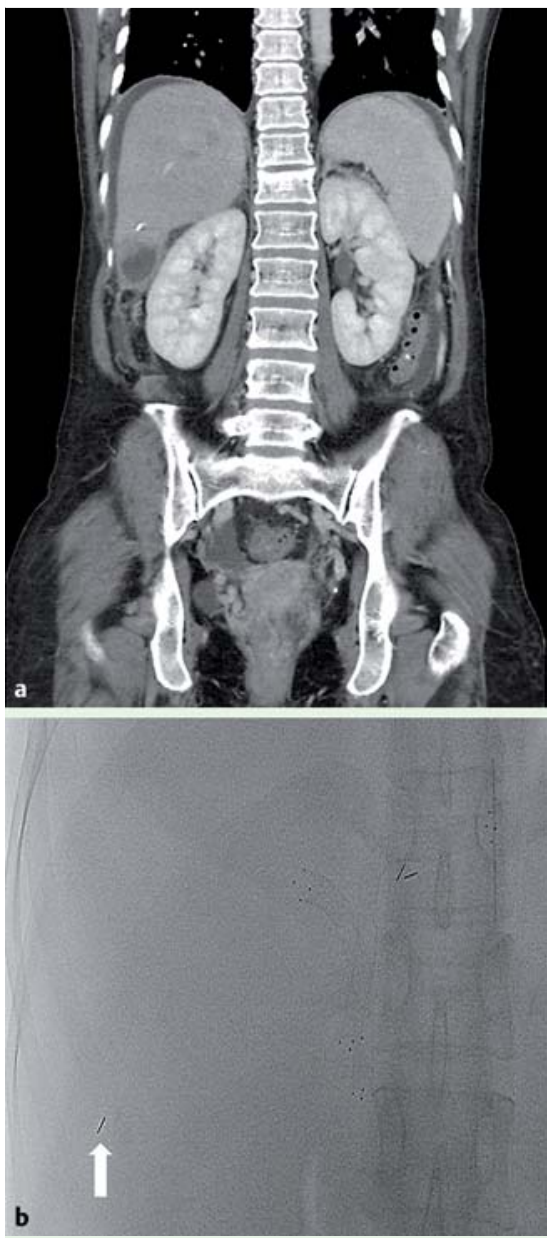

Fig. 3 a, b A computed tomography (CT) scan 6 months after radiation therapy revealed a hepatic infarction at $\mathrm{S} 6$ because of right portal vein occlusion as a result of migration of a fiducial marker to the right portal vein.
Spontaneous fiducial migration is a relatively rare occurrence as are migrationrelated complications [1,2]. Therapeutic data for a hepatic infarction caused by spontaneous fiducial migration are limited, and our case was resolved with conservative care. This case may represent the first report of a hepatic infarction caused by spontaneous fiducial migration.

\section{Endoscopy_UCTN_Code_CPL_1AL_2AG}

\section{Competing interests: None}

Dongwook Oh, Do Hyun Park, Young Whan Cho, Tae Jun Song, Sang Soo Lee, Dong Wan Seo, Sung Koo Lee, Myung-Hwan Kim

Division of Gastroenterology, Department of Internal Medicine, University of Ulsan College of Medicine, Asan Medical Center, Seoul, Korea

\section{References}

1 Choi JH, Seo DW, Park doH et al. Fiducial placement for stereotactic body radiation therapy under only endoscopic ultrasonography guidance in pancreatic and hepatic malignancy: practical feasibility and safety. Gut Liver 2014; 8: 88-93

2 Sanders MK, Moser AJ, Khalid A et al. EUSguided fiducial placement for stereotactic body radiotherapy in locally advanced and recurrent pancreatic cancer. Gastrointest Endosc 2010; 71: 1178-1184

\section{Bibliography}

Dol http://dx.doi.org/

10.1055/s-0034-1377771

Endoscopy 2014; 46: E585-E586

(c) Georg Thieme Verlag KG

Stuttgart · New York

ISSN 0013-726X

\section{Corresponding author}

\section{Do Hyun Park, MD, PhD}

Division of Gastroenterology

Department of Internal Medicine

University of Ulsan College of Medicine

Asan Medical Center

88, Olympic-Ro 43-Gil

Songpa-gu

Seoul 138-736

Korea

Fax: +82-2-4760824

dhpark@amc.seoul.kr 